\title{
RELIABLE CELL TRACKING BY GLOBAL DATA ASSOCIATION
}

\author{
Ryoma Bise, Zhaozheng Yin, and Takeo Kanade \\ Carnegie Mellon University, Pittsburgh, PA, USA.
}

\begin{abstract}
Automated cell tracking in populations is important for research and discovery in biology and medicine. In this paper, we propose a cell tracking method based on global spatiotemporal data association which considers hypotheses of initialization, termination, translation, division and false positive in an integrated formulation. Firstly, reliable tracklets (i.e., short trajectories) are generated by linking detection responses based on frame-by-frame association. Next, these tracklets are globally associated over time to obtain final cell trajectories and lineage trees. During global association, tracklets form tree structures where a mother cell divides into two daughter cells. We formulate the global association for tree structures as a maximum-a-posteriori (MAP) problem and solve it by linear programming. This approach is quantitatively evaluated on sequences with thousands of cells captured over several days.
\end{abstract}

Index Terms - cell tracking, global data association

\section{INTRODUCTION}

Analysis of stem cell behaviors in populations is important for research and discovery in biology and medicine. To obtain the quantitative measurement of cell behaviors, time-lapse microscopy videos consisting of hundreds of cells over thousands of frames are recorded to track each cell's dynamic process. It is very time consuming for human to analyze this huge amount of data manually, thus automated cell tracking is required to meet this demand.

To analyze stem cell behaviors, many cell tracking methods have been proposed which can be roughly classified into two groups: model-based contour evolution approaches and segmentation-based data association approaches. The modelbased contour evolution approaches, in particular level-set methods, are widely used in cell segmentation and tracking [1][2]. The tracking is performed by finding the object contour in the current frame given an initial contour from the previous frame. While these methods can handle changes in topology, it is hard to handle the dividing cells, cells that enter/leave the field of view. To handle these cases, Li et al. [2] associate new cells with its parent cell as daughter cells by using a local association method.

Segmentation-based frame-by-frame association approaches have also been shown to be effective for cell tracking [3][4][5]. In [3], Al-Kofahi et al. segment the cell regions using an adaptive thresholding method, and then, resolve the association between two frames by optimizing probabilistic objective functions based on distance measures. The authors mentioned that in some cases, multiple cells merge into a cluster and eventually split apart, making tracking difficult, and they did not address cells leaving or entering the field of view. In [4], Padfield et al. use the graph theoretic minimum cost flow framework to resolve the data association in which the hypotheses include various cell behaviors such as migration, mitosis, overlap, entering and leaving. The method achieved high accuracy in tracking and detecting cell behaviors for the experiments. However, the method may confuse the cell identities when cells touch or overlap for long frames. To resolve the case in which cells overlapped for long frames, in [5], Bise et al. proposed the contour tracking method based on partial contour matching. The method firstly detects a cluster in which multiple cells touch or overlap, and it then separates the cell contour as its member cells. The separated cells maintain their identities for the association problems in the following frames.

These frame-by-frame association methods achieved high tracking accuracy based on trajectory-level evaluation (how well ground-truth cells are followed by computer-generated tracks). However, it is still challenging to achieve high accuracy based on lineage-level (tree structure) evaluation including the correctness of the mother-daughter relationship. For example, when a false positive segmentation appears near a mitotic cell, the local temporal association methods may cause a mother-daughter relationship error. To resolve the problem, global temporal information is important. If we observe the cells for several frames after the birth event, we can easily recognize that one of the children cells is false positive since false positives usually disappear soon. This allows us to correct the relationship.

Recently, global spatio-temporal data association approaches have been proposed for the general object tracking. To associate multiple trajectories over time, Multi-Hypothesis Tracking (MHT) [6] and Joint Probabilistic Data Association Filters (JPDAF)[7] are two representative examples. To reduce the computational cost, tracklet stitching [8] is proposed. Huang et al. [8] first generate reliable tracklets that are fragments of tracks formed by conservative grouping of detection 


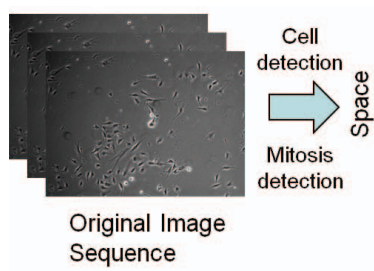

Sequence
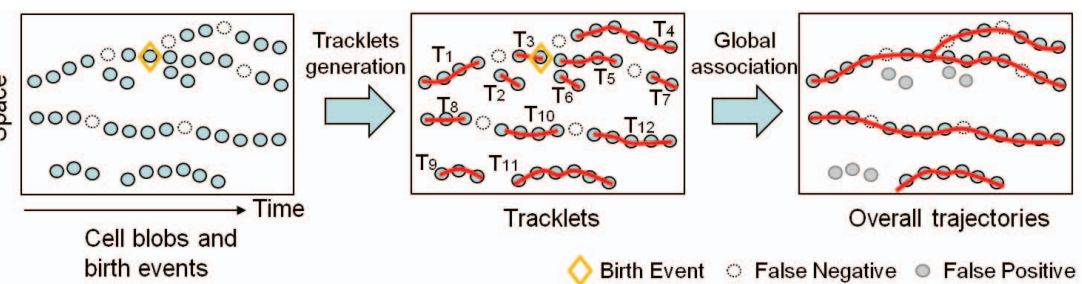

Overall trajectories

birth events

Fig. 1. System Overview.

responses. The tracklets are then connected by the Hungarian algorithm [9]. Bonneau et al. [10] proposed a tracklets linking method in which a minimal path between tracklets is obtained by using dynamic programming in order to track quantum dots in a living cell. Zhang et al. [11] proposed a minimum-cost flow network to resolve the global data association of multiple objects over time. These global association approaches are known as achieving higher accuracy of tracking general objects than frame-by-frame association methods. However, these approaches cannot be applied to cell tracking directly, since they do not consider cell division (a mother cell divides into two daughter cells to form a tree structure in the trajectory).

In this paper, we propose a global spatio-temporal data association method for the tree structure to obtain cell trajectories and lineage trees. Reliable tracklets (i.e., short trajectories) are firstly generated by linking detection responses based on frame-by-frame association. The global tracklet association for the tree structures is then formulated as a maximuma-posteriori (MAP) problem. The MAP problem is solved by a linear programming to provide the cell trajectories and lineage trees. This approach is evaluated on five sequences with thousands of cells captured over several days. The results show an improvement of the tracking performance compared to our previous method [2] that used the level-set technique for tracking.

\section{ALGORITHM}

Fig. 1 shows the overview of our cell tracking method. First, cell detection module segments cell blobs from input images that may include false positives and false negatives, and mitosis detection module locates birth events where and when one cell divides into two cells. Next, the detected cell blobs are associated to reliable tracklets by a frame-by-frame data association. Finally, the global association module associates the tracklets to obtain cell trajectories and lineage trees.

\subsection{Cell Detection}

Due to the interference optics of a phase contrast microscope, cells are surrounded by bright halos, and cellular fluid inside the membrane has similar intensity as the background. To facilitate segmentation, we have adopted the image restoration technique recently developed in [12]. The technique utilizes

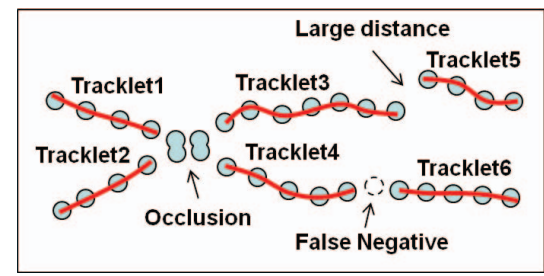

Fig. 2. Examples of tracklets.

the optophysical principle of image formation by phase contrast microscope, and transforms an input image to an artifactfree image by minimizing a regularized quadratic cost function. In the restored image, cells appear as regions of positive values against a uniformly-zero background. A simple thresholding method, such as Otsu thresholding, can segment out the cell regions. We denote the set of detection results as $\mathbf{R}=\left\{R_{i}\right\}$ where $R_{i}$ represents the $i$ th cell blob.

\subsection{Cell Mitosis Event Detection}

To detect the birth events (time and location at which one cell divides into two cells), we have adopted the mitosis detection technique recently developed in [13]. Firstly, as a mitosis event generally exhibits an increase of brightness, bright regions are extracted as patches, and then candidate patch sequences are constructed by associated patches. Next, the gradient histogram features are extracted from the patches. Finally, a probabilistic model named Event Detection Conditional Random Field (EDCRF) is applied to determine whether each candidate patch sequence contains a birth event and which frame the birth event is located in. The set of the detected mitosis events is represented as $\mathbf{M}=\left\{M_{i}\right\}$ where $M_{i}$ is a detected mitosis event.

\subsection{Tracklet Generation}

Since a long trajectory obtained via frame-by-frame association may include more failures, such as drift and occlusion, than a short trajectory, we firstly associate the detected blobs to make reliable tracklets. A tracklet is considered reliable when cell blobs in consecutive frames are close enough, and there are no extra confusing blobs near the cell. Fig. 2 shows examples of extracted tracklets in which two cells migrate. In this example, when the occlusions occur, it is not clear if a detection response right after the occlusion is associated with tracklet 1 or 2 , so tracklet 1 and 2 are terminated at that time. False negatives and large distance between the blobs 


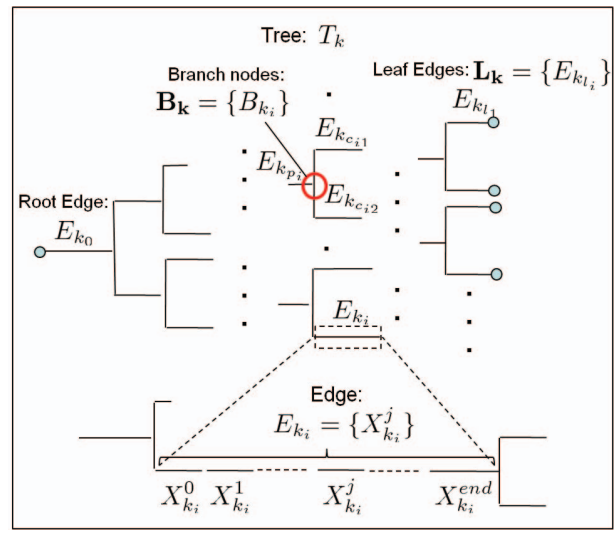

Fig. 3. An example of a tree structure hypothesis. Bottom illustration shows zooming of an edge which consists of tracklets.

also cause uncertain association, so tracklets 3 and 4 are also terminated at that time.

For the implementation, we use a frame-by-frame association method to generate tracklets. The cell association algorithm makes hypotheses of all possible cell translation and computes their likeliness as:

$$
P_{\text {link }}\left(b_{j} \mid c_{i}\right)=e^{-\frac{\left\|f\left(c_{i}\right)-f\left(b_{j}\right)\right\|}{\sigma}}
$$

where $c_{i}$ represents the $i$ th cell in the previous frame and $b_{j}$ represents the $j$ th blob in the current frame. $f(\cdot)$ computes an object's feature vector where different types of features can be incorporated such as appearance histogram, shape and motion history. Then, the optimal association from the hypothesis set is found by solving an integer optimization problem which is similar to an optimization approach used by [2] for track linking. The detected blob is linked to a cell if and only if their likeliness is higher than a threshold.

Based on the frame-by-frame association, we generate a set of reliable tracklets $\mathbf{X}=\left\{X_{i}\right\} . X_{i}=\left\{R_{i_{j}}\right\}$ is a tracklet consisting of an order list of detection results where $R_{i_{j}}$ indicates the $j$ th detection result on the tracklet $X_{i}$. Any isolated detection response that is not linked with any other one is considered as a tracklet and also included in $\mathbf{X}$. Unclear associations are solved on the next step by using the global data association.

\subsection{Global Data Association}

In this section, we propose a global data association method which addresses the tree structure association problem.

Let $\mathbf{T}=\left\{T_{k}\right\}$ be a hypotheses set of cell trajectory trees over the entire video. Each tree $T_{k}$, corresponding to a cell family from the ancestor to all of its descendents, is formed by associated tracklets. We define a tree structure hypothesis on $T_{k}$ using the following notations (Fig. 3):

1. $\mathbf{E}_{\mathbf{k}}=\left\{E_{k_{i}}\right\}$ : a set of edges of the tree $T_{k}$. Each edge is defined as an order list of tracklets, i.e., $E_{k_{i}}=\left\{X_{k_{i}}^{j}\right\}$ where $X_{k_{i}}^{j}$ is $j$ th tracklet on the edge $E_{k_{i}}$. Specifically, $E_{k_{0}}$ denotes the root edge of the tree.

2. $\mathbf{B}_{\mathbf{k}}=\left\{B_{k_{i}}\right\}$ : a set of branch nodes of tree $T_{k}$. Each branch node $B_{k_{i}}$ defines a parent-children relationship, $B_{k_{i}}=\left\{E_{k_{p_{i}}}, E_{k_{c_{i 1}}}, E_{k_{c_{i 2}}}\right\}\left(E_{k_{p_{i}}}\right.$ is a parent, and $E_{k_{c_{i 1}}}, E_{k_{c_{i 2}}}$ are children.)

3. $\mathbf{L}_{\mathbf{k}}=\left\{E_{k_{l_{i}}}\right\}$ : a set of leaf edges of tree $T_{k}$.

Given the observation tracklet set $\mathbf{X}$, we maximize the posteriori probability to solve for the best hypothesis $\mathbf{T}^{*}$.

$$
\begin{aligned}
\mathbf{T}^{*} & =\arg \max _{\mathbf{T}} P(\mathbf{T} \mid \mathbf{X}) \\
& =\arg \max _{\mathbf{T}} P(\mathbf{X} \mid \mathbf{T}) P(\mathbf{T}) \\
& =\arg \max _{\mathbf{T}} \prod_{X_{i} \in \mathbf{X}} P\left(X_{i} \mid \mathbf{T}\right) \prod_{T_{k} \in \mathbf{T}} P_{\text {Tree }}\left(T_{k}\right)
\end{aligned}
$$

In Eq. 1, we assume that the likelihoods of input tracklets are conditionally independent given $\mathbf{T}$, and $T_{k} \in \mathbf{T}$ can not overlap with each other, i.e., $T_{k} \cap T_{l}=\phi, \forall k \neq l$. The likelihood of observed tracklet $X_{i}$ is

$$
P\left(X_{i} \mid \mathbf{T}\right)=\left\{\begin{array}{l}
P_{T P}\left(X_{i}\right), \text { if } \exists T_{k} \in \mathbf{T}, X_{i} \in T_{k} \\
P_{F P}\left(X_{i}\right), \text { otherwise }
\end{array}\right.
$$

where $P_{T P}\left(X_{i}\right)$ is the probability for $X_{i}$ being a true positive, and $P_{F P}\left(X_{i}\right)$ is the probability for $X_{i}$ being a false alarm. $P_{\text {tree }}\left(T_{k}\right)$ is modeled as a Markov chain:

$$
\begin{array}{r}
P_{\text {Tree }}\left(T_{k}\right)=P_{\text {ini }}\left(E_{k_{0}}\right) \times \prod_{E_{k_{i}} \in T_{k}} P_{\text {edge }}\left(E_{k_{i}}\right) \\
\left.\quad \times \prod_{\left\{E_{k_{p_{i}}}, E_{k_{c_{i 1}}}, E_{k_{c_{i 2}}}\right\} \in \mathbf{B}_{\mathbf{k}}, \mathbf{B}_{\mathbf{k}} \in T_{k}} P_{k_{c_{i 1}}}, E_{k_{c_{i 2}}} \mid E_{k_{p_{i}}}\right) \\
\quad \times \prod_{E_{k_{l_{i}}} \in \mathbf{L}_{\mathbf{k}}, \mathbf{L}_{\mathbf{k}} \in T_{k}} P_{\text {term }}\left(E_{k_{l_{i}}}\right)
\end{array}
$$

where $P_{i n i}$ is an initialization probability on the root of the tree, and $P_{\text {term }}$ is a termination probability on a leaf of the tree. $P_{d i v}\left(E_{k_{c_{i 1}}}, E_{k_{c_{i 2}}} \mid E_{k_{p_{i}}}\right)$ is an edge dividing probability in which edge $E_{k_{p_{i}}}$ divides into two edges $E_{k_{c_{i 1}}}, E_{k_{c_{i 2}}}$. Under the Markov assumption, the edge probability can be formulated as:

$$
P_{\text {edge }}\left(E_{k_{i}}\right)=\prod_{j=1: N_{k_{i}}-1} P_{\text {link }}\left(X_{k_{i}}^{j} \mid X_{k_{i}}^{j-1}\right)
$$

where $P_{\text {link }}\left(X_{k_{i}}^{j} \mid X_{k_{i}}^{j-1}\right)$ is the probability to link tacklets $X_{k_{i}}^{j}$ and $X_{k_{i}}^{j-1}$ together, $N_{k_{i}}$ is the number of tracklets on the edge $E_{k_{i}}$. Let $X_{k_{i}}^{0}$ be the first tracklet of $E_{k_{i}}$, and $X_{k_{i}}^{\text {end }}$ be the last tracklet of $E_{k_{i}}$. Under the Markov assumption, the initialization, termination, and dividing probabilities can be formulated as:

$$
\begin{array}{r}
P_{i n i}\left(E_{k_{0}}\right)=P_{i n i}\left(X_{k_{0}}^{0}\right), \\
P_{\text {term }}\left(E_{k_{l_{i}}}\right)=P_{\text {term }}\left(X_{k_{l_{i}}}^{e n d}\right), \\
P_{d i v}\left(E_{k_{c_{i 1}}}, E_{k_{c_{i 2}}} \mid E_{k_{p_{i}}}\right)=P_{d i v}\left(X_{k_{c_{i 1}}}^{0}, X_{k_{c_{i 2}}}^{0} \mid X_{k_{p_{i}}}^{\text {end }}\right)
\end{array}
$$


After substituting Eqs. 2-7 into Eq. 1, we take a logarithm on the objective function:

$$
\begin{aligned}
& \mathbf{T}^{*}=\arg \max _{\mathbf{T}}\left\{\sum_{X_{i} \notin T_{k}, \forall T_{k} \in \mathbf{T}} \log P_{F P}\left(X_{i}\right)\right. \\
& +\sum_{X_{i} \in T_{k}, \forall T_{k} \in \mathbf{T}} \log P_{T P}\left(X_{i}\right) \\
& +\sum_{X_{k_{0}}^{0} \in E_{k_{0}},} \sum_{E_{k_{0}} \in T_{k}, \forall T_{k} \in \mathbf{T}} \log P_{\text {ini }}\left(X_{k_{0}}^{0}\right) \\
& +\sum_{X_{k_{i}}^{j}, X_{k_{i}}^{j-1} \in E_{k_{i}}, \forall E_{k_{i}} \in T_{k}, \forall T_{k} \in \mathbf{T}} \log P_{l i n k}\left(X_{k_{i}}^{j} \mid X_{k_{i}}^{j-1}\right) \\
& +\sum_{\left\{X_{k_{p_{i}}}^{\text {end }}, X_{k_{c_{i 1}}}^{0}, X_{k_{c_{i 1}}}^{0}\right\} \in \mathbf{B}_{\mathbf{k}}, \mathbf{B}_{\mathbf{k}} \in T_{k}, \forall T_{k} \in \mathbf{T}} \log P_{\text {div }}\left(X_{k_{c_{i 1}}}^{0}, X_{k_{c_{i 2}}}^{0} \mid X_{k_{p_{i}}}^{e n d}\right) \\
& \left.+\sum_{X_{k_{l_{i}}}^{\text {end }} \in E_{k_{l_{i}}}, \forall E_{k_{l_{i}}} \in \mathbf{L}_{\mathbf{k}}, \mathbf{L}_{\mathbf{k}} \in T_{k}, \forall T_{k} \in \mathbf{T}} \log P_{\text {term }}\left(X_{k_{l_{i}}}^{\text {end }}\right)\right\}
\end{aligned}
$$

The above MAP problem is solved by linear programming. Let $N_{\mathbf{X}}$ be the number of tracklets in the entire sequence, vector $\rho$ stores the likelihoods of every possible hypothesis and matrix $C$ stores the constraints to avoid conflict hypotheses, where each row of $C$ has $2 N_{\mathbf{X}}$ columns and each column indicates tracklet index on the association between two tracklets. We compute the entries of $\rho$ and $C$ based on the following hypotheses.

1. Initialization hypothesis:

If the first blob of a tracklet $X_{k}$ appears in the beginning of the sequence or appears near the boundary of the field of view, the tracklet is a candidate of a initial tracklet. Let $h$ be the index of a new hypothesis, we append a new row to $C$ and a corresponding likelihood to $\rho$ :

$$
\begin{aligned}
C(h, i) & = \begin{cases}1, & \text { if } i=N_{\mathbf{X}}+k \\
0, & \text { otherwise. }\end{cases} \\
\rho(h) & =\log P_{i n i}\left(X_{k}\right)+0.5 \log P_{T P}\left(X_{k}\right)
\end{aligned}
$$

2. Termination hypothesis:

If the last blob of a tracklet $X_{k}$ appears in the end of the sequence or appears near the boundary of the field of view, the tracklet is a candidate of a termination tracklet. We define new entries for $C$ and $\rho$ as:

$$
\begin{aligned}
C(h, i) & = \begin{cases}1, & \text { if } i=k \\
0, & \text { otherwise. }\end{cases} \\
\rho(h) & =\log P_{\text {term }}\left(X_{k}\right)+0.5 \log P_{T P}\left(X_{k}\right)
\end{aligned}
$$

3. Translation hypothesis:

If the time and space distances between the last blob of tracklets $X_{k_{1}}$ and the first blob of $X_{k_{2}}$ are less than thresholds, $X_{k_{1}} \rightarrow X_{k_{2}}$ is a candidate of a tracklet

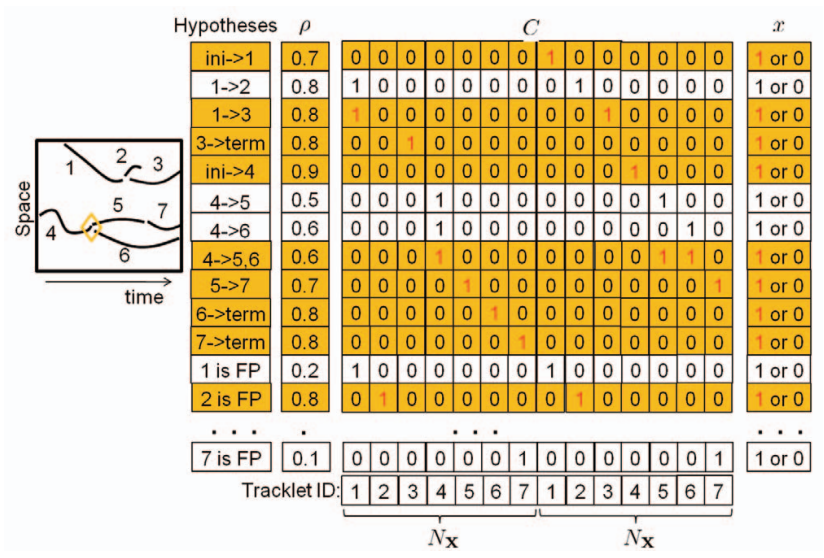

Fig. 4. An integer programming example where the optimal solution is highlighted by orange.

translation. We define new entries for $C$ and $\rho$ as:

$$
\begin{aligned}
C(h, i) & =\left\{\begin{array}{l}
1, \text { if } i=k_{1} \text { or } i=N_{\mathbf{X}}+k_{2} \\
0, \text { otherwise. }
\end{array}\right. \\
\rho(h) & =\log P_{l i n k}\left(X_{k_{2}} \mid X_{k_{1}}\right) \\
& +0.5 \log P_{T P}\left(X_{k_{1}}\right)+0.5 \log P_{T P}\left(X_{k_{2}}\right)
\end{aligned}
$$

4. Dividing hypothesis:

If the last blob of a tracklet $X_{p}$ is near a birth event detected by mitosis detection module, the tracklet is a candidate of the parent tracklet, and if the first blobs of some other tracklets $X_{c 1}, X_{c 2}$ are near the candidate parent tracklet, these tracklets are candidates of the children tracklets, we define new entries for $C$ and $\rho$ as:

$$
\begin{aligned}
C(h, i) & =\left\{\begin{array}{c}
1, \text { if } i=p \text { or } i=N_{\mathbf{X}}+c_{1}, \\
\text { or } i=N_{\mathbf{X}}+c_{2} \\
0, \text { otherwise. }
\end{array}\right. \\
\rho(h) & =\log P_{d i v}\left(X_{c_{1}}, X_{c_{2}} \mid X_{p}\right)+0.5 \log P_{T P}\left(X_{p}\right) \\
& +0.5 \log P_{T P}\left(X_{c_{1}}\right)+0.5 \log P_{T P}\left(X_{c_{2}}\right)
\end{aligned}
$$

5. False positive hypothesis:

All of the tracklets can be false positive. When $X_{k}$ is a candidate of a false positive on the hypothesis $h$, we define new entries for $C$ and $\rho$ as:

$$
\begin{aligned}
C(h, i) & =\left\{\begin{array}{l}
1, \text { if } i=k \text { or } i=N_{\mathbf{X}}+k \\
0, \text { otherwise. }
\end{array}\right. \\
\rho(h) & =\log P_{F P}\left(X_{k}\right)
\end{aligned}
$$

A true positive tracklet appears in two and only two associations in the optimal solution: the first blob of the tracklet appears in an initialization, translation or dividing hypothesis, and the last blob of the tracklet appears in a translation, dividing or termination hypothesis. Thus, $\log P_{T P}\left(X_{k}\right)$ in the second term of Eq. 8 is divided into two halves that are integrated into the two neighboring transition hypotheses respectively, as described in hypotheses 1-4. 
After making $M$ hypotheses over $N_{\mathbf{X}}$ tracklets, the MAP problem in Eq. 8 can be considered as selecting a subset of rows of $C$ such that the sum of corresponding elements in $\rho$ is maximized, under the constraint where any trees can not overlap with each other. This can be formulated as the following integer optimization problem:

$$
x^{*}=\arg \max _{x} \rho^{T} x, \quad \text { s.t. } C^{T} x=1
$$

where $x$ is a $M \times 1$ binary vector, and $x_{k}=1$ means the $k$ th hypothesis is selected in the global optimal solution. The constraint $C^{T} x=1$ guarantees that each tracklet ID appears in only one associated tree or false positive tracklet. Fig. 4 shows a simple example of the linear programming where the number of tracklets is 7 and the number of hypotheses is 18 . In the optimal solution, initial tracklet 1 is linked to tracklet 3 (i.e., tracklets 1, 3 are associated as a single edge tree). Initial tracklet 4 divides into 5 and 6 , tracklet 5 is linked to tracklet 7 , and tracklets 6 and 7 are termination tracklets (i.e., tracklets 4, 5, 6 and 7 are associated as a binary tree).

\subsection{Implementation Details}

In this section, we describe the estimation of the probabilities in our framework. Let $\alpha$ be the miss detection rate of the cell detector, and $\left|X_{i}\right|$ be the number of total detection responses in a tracklet $X_{i}$. The probabilities of false positive and true positive are defined as:

$$
\begin{array}{r}
P_{F P}\left(X_{i}\right)=\alpha^{\left|X_{i}\right|} \\
P_{T P}\left(X_{i}\right)=1-P_{F P}\left(X_{i}\right)
\end{array}
$$

The initialization probability is defined based on the time distance between the beginning of the sequence and the first appearance frame of the tracklet, or the spatial distance between the boundary of the field of view and the cell centroid for the cell entering case.

$$
P_{i n i}\left(X_{i}\right)=\left\{\begin{array}{cl}
e^{-\frac{d t_{0}\left(R_{i_{0}}\right)}{\lambda_{1}}}, & \text { if } d t_{0}\left(R_{i_{0}}\right)<\theta_{t} \\
e^{-\frac{d s\left(R_{i_{0}}\right)}{\lambda_{2}}}, & \text { if } d s\left(R_{i_{0}}\right)<\theta_{s} \\
\eta & \text { otherwise }(\eta \text { is small })
\end{array}\right.
$$

where $R_{i_{0}}$ is the first detection response of the tracklet $X_{i}$, $d t_{0}\left(R_{i}\right)$ is the time distance between the first frame of the sequence and the frame when the detection response $R_{i}$ appears. $d s\left(R_{i}\right)$ is the distance between the centroid of the detection response $R_{i}$ and the image boundary. $\lambda_{1}$ and $\lambda_{2}$ are free parameters to adjust the distribution. If the first detection response of the tracklet appears in both beginning of the sequence and near the boundary, we take a maximam one for the probability.

The termination probability is defined in a similar way as the initialization probability.

$$
P_{\text {term }}\left(X_{i}\right)=\left\{\begin{array}{cl}
e^{\left.-\frac{d t_{e n d}\left(R_{i_{e n d}}\right)}{\lambda_{1}}\right)}, \text { if } d t_{\text {end }}\left(R_{i_{\text {end }}}\right)<\theta_{t} \\
e^{-\frac{d s\left(R_{i_{e n d}}\right)}{\lambda_{2}},}, \text { if } d s\left(R_{i_{\text {end }}}\right)<\theta_{s} \\
\eta, & \text { otherwise }(\eta \text { is small })
\end{array}\right.
$$
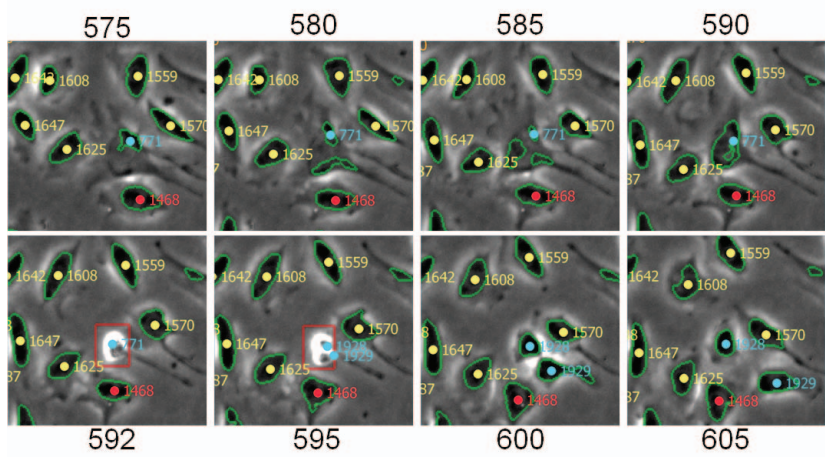

Fig. 5. Example images of tracking results. Green contours are segmented cell boundaries. Red color boxes are detected mitosis events. The numbers in the images are cell ID. The number on the top and bottom of the images are frame indexes. The colors of cell IDs indicate their family identity. Cells with the same color have the same ancestor.

where $R_{i_{\text {end }}}$ is the last detection response of the tracklet $X_{i}$, $d t_{\text {end }}\left(R_{i}\right)$ is the time distance between the last frame of the sequence and the frame when the detection response $R_{i}$ appears.

The link probability between two tracklets and the dividing probability that one tracklet divides to two tracklets are defined as:

$$
\begin{aligned}
& P_{\text {link }}\left(X_{j} \mid X_{i}\right)=e^{\left.-\left\|g\left(R_{j_{0}}\right)-g\left(R_{i_{e n d}}\right)\right\|\right) / \lambda_{3}} \\
& P_{d i v}\left(X_{c 1}, X_{c 2} \mid X_{p}\right) \\
& \quad=e^{-\left(\left\|g\left(R_{p_{e n d}}\right)-g\left(R_{c 1_{0}}\right)\right\|+\left\|g\left(R_{p_{e n d}}\right)-g\left(R_{c 2_{0}}\right)\right\|\right) / 2 \lambda_{3}}
\end{aligned}
$$

where $g(\cdot)$ computes an object's feature vector in which different types of features can be incorporated such as appearance time and motion history. $\lambda_{3}$ is a free parameter to adjust the distribution. Based on the cell movement history, we set these parameter as: $\lambda_{1}=5, \lambda_{2}=30, \lambda_{3}=25, \theta_{t}=15$, $\theta_{s}=40$.

\section{EXPERIMENTAL RESULTS}

\subsection{Tracking Results}

Fig. 5 shows an example sequence of tracking results. The cell 771 on the center of the image spreads out and the boundary is ambiguous, thus, from frame 580 to 585 , the cell are segmented to multiple regions some of which are false positives. These false positives disappear in several frames, and only one region can be associated with the tree. Since our global association framework uses not only space and appearance information but also temporal information, our method tracks the cells well and recognizes the false positives. Using the detected mitosis event information (red box in Fig. 5), the proposed method makes a hypothesis of cell division, thus, the two children cells 1928 and 1929 are correctly associated to the parent cell 771 . 

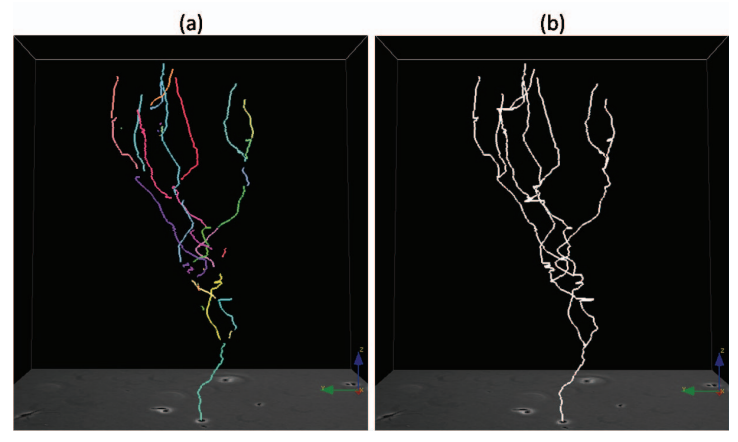

Fig. 6. An example of space-time trajectory plot of a cell family. (a) Tracklets. (b) A tree in which tracklets are associated by global data association.
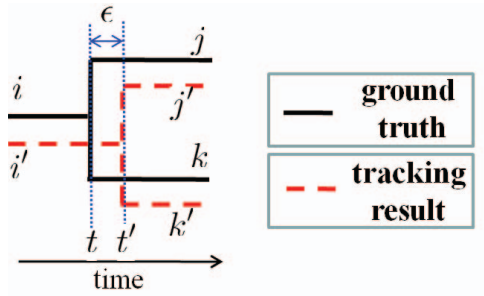

Fig. 7. Evaluation of mitosis branching.

Fig. 6(a) shows the tracklets before the global association and Fig. 6(b) shows the associated tree after the global association. There are 38 tracklets in Fig. 6(a) including false positives and false negatives. The true positive tracklets are well associated to a tree and false positive tracklets are removed by global data association as shown in Fig. 6(b).

\subsection{Quantitative evaluation}

\subsubsection{Data}

We recorded five sequences captured at the resolution of $1040 \times 1392$ pixels where $\mathrm{C} 2 \mathrm{C} 12$ muscle stem cells growing from $30+$ to $600+$ are imaged every 5 minutes by ZEISS Axiovert $135 \mathrm{TV}$ phase contrast microscope at $5 \mathrm{X}$ magnification over 65 hours (780 images). For one image sequence, all cells are annotated. Since it is extremely time-consuming to annotate all cells, for the other four image sequences, three cells are randomly selected in the initial frame and their progeny cells are manually tracked. The total number of annotated cells in the five sequences is 124621 .

\subsubsection{Metrics}

We use three quantitative criteria to assess the system performance: track purity, target effectiveness [14], and mitosis branching correctness.

To compute target effectiveness, we first assign each target (human annotated) to a track (computer-generated) that contains the most observations from that ground-truth. Then target effectiveness is computed as the number of the assigned track observations over the total number of frames of the target. It indicates how many frames of targets are followed by computer-generated tracks. Similarly, we define track purity as how well tracks are followed by targets.

\begin{tabular}{|c|c|c|c|}
\hline & $\begin{array}{c}\text { Track } \\
\text { Purity }\end{array}$ & $\begin{array}{c}\text { Target } \\
\text { Effectiveness }\end{array}$ & $\begin{array}{c}\text { Mitosis Branching } \\
\text { Correctness }\end{array}$ \\
\hline Li et al. [2] & 0.62 & 0.70 & 0.46 \\
\hline Ours & 0.81 & 0.87 & 0.65 \\
\hline
\end{tabular}

Table 1. Comparison of our system with [2] on a sequence with all cells annotated.

\begin{tabular}{|c|c|c|c|c|}
\hline \multirow{2}{*}{} & \multicolumn{2}{|c|}{$\begin{array}{c}\text { Target } \\
\text { Effectiveness }\end{array}$} & \multicolumn{2}{c|}{$\begin{array}{c}\text { Mitosis Branching } \\
\text { Correctness }\end{array}$} \\
\cline { 2 - 5 } & ours & Li et al. & ours & Li et al \\
\hline exp1 & 0.96 & 0.75 & 0.75 & 0.25 \\
\hline exp2 & 0.87 & 0.7 & 0.65 & 0.63 \\
\hline exp3 & 0.87 & 0.68 & 0.59 & 0.39 \\
\hline exp4 & 0.78 & 0.6 & 0.57 & 0.2 \\
\hline average & 0.87 & 0.68 & 0.64 & 0.37 \\
\hline
\end{tabular}

Table 2. Comparison of our system with [2] on four sequences.

The mitotic branching correctness measured the accuracy of mother-daughter relationships between tree branches. Fig. 7 shows an example of a mitosis branch, black lines indicate ground truth trajectory, and red dotted lines indicate tracking results. In the ground truth, there is a birth event at time $t$ in which cell $i$ divides into cell $j$ and $k$. If the automatic tracking results include a birth event of the cell $i^{\prime}$ that corresponds to cell $i$, and children $j^{\prime}, k^{\prime}$ of the cell $i^{\prime}$ are also corresponded to cell $j$ and $k$, and the time distance between the two birth events, $\epsilon=\left\|t-t^{\prime}\right\|$, is close enough (i.e., $\epsilon<\theta_{\epsilon}$ ), it is considered as a correctly detected mitosis branching. The correctness of mitotic branching is the number of the correctly detected mitosis branching over the total number of the mitotic events. In the evaluation, we set the parameter as $\theta_{\epsilon}=10$.

\subsubsection{Performance evaluation}

Fig. 8 shows an example image of cell tracking with track IDs and segmented regions. Cells are well segmented and tracked in the population. Fig. 9 shows the space-time trajectory plot of the whole sequence. It represents the complete history of the cell population: motions of all the cells and their lineage information. Fig. 10 shows examples of the lineage trees compared to human annotated ones. Horizontal red lines indicate tracks that follow the ground-truth, vertical red lines indicate that the mitosis branching is correctly detected on the branch nodes of the lineage tree. The results show that the lineage trees are well constructed.

As shown in Table 1, our system achieves higher accuracy on all of the performance metrics than the method presented in [2] on the full-annotated sequence. Table 2 summarizes the target effectiveness and mitotic branching correctness comparison on four image sequences ${ }^{1}$. On average, we improved $19 \%$ on target effectiveness and $27 \%$ on mitosis branching correctness compared with [2].

\footnotetext{
${ }^{1}$ We are not able to compute track purity for the four partially-annotated sequences because it needs all cells to be annotated.
} 


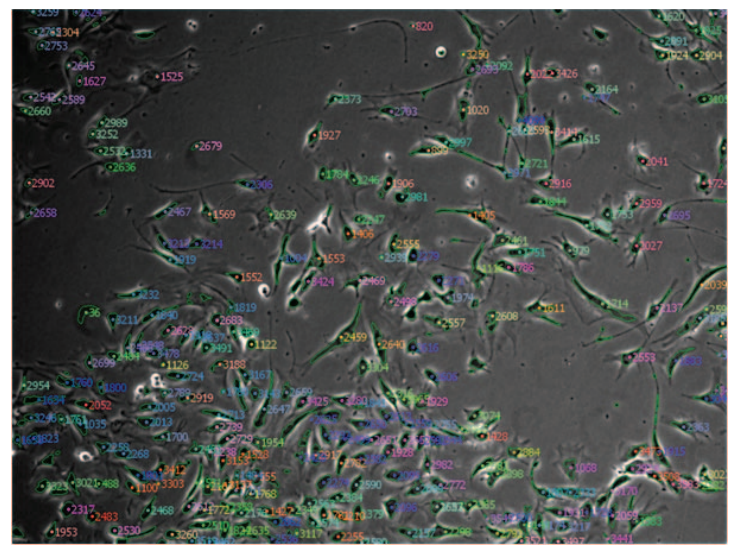

Fig. 8. An example of tracking result image with track IDs and segmented regions.

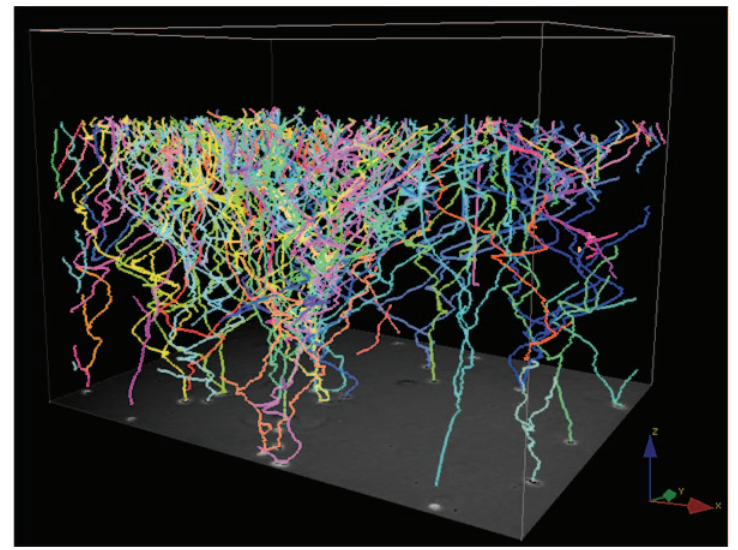

Fig. 9. A space-time trajectory plot of the whole sequence. $X$ and $\mathrm{Y}$ axes represent $2 \mathrm{D}$ space, $\mathrm{Z}$ axis represents time.

\section{CONCLUSION}

We proposed a global data association framework for cell tracking problem. The proposed method can associate tracklets to form not only sequential structures but also tree structures. The results of the data association provide the full cell trajectories and lineage trees. Experimental results on a challenging data set show that the proposed method significantly improves the tracking performance including target effectiveness, track purity, mitosis branching correctness by globally associating tracklets.

\section{REFERENCES}

[1] F. Yang, M. Mackey, F. Ianzini, G. Gallardo, and M. Sonka, "Cell segmentation, tracking, and mitosis detection using temporal context," MICCAI, 302-309, 2005.

[2] K. Li, E.D. Miller, M. Chen, T. Kanade, L.E.Weiss, and P.G. Campbell, "Cell population tracking and lineage construction with spatiotemporal context," Med Image Anal, 12(5), 546-566, 2008.

[3] O. Al-Kofahi, R. Radke, S. Goderie, Q. Shen, S. Temple,

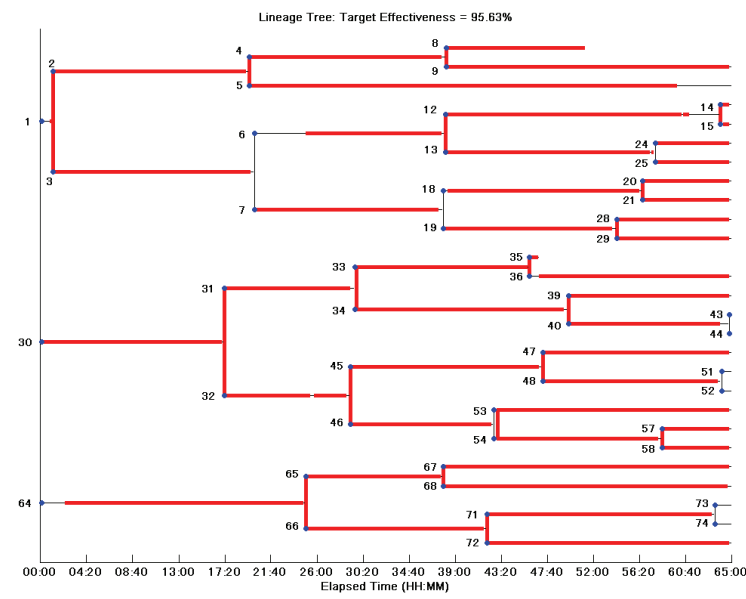

Fig. 10. Lineage trees and performance evaluation (thin black lines: three human annotated lineage trees; overlaid thick red lines: correctly-tracked cells by our system).

and B. Roysam, "Automated cell lineage construction: A rapid method to analyze clonal development established with murine neural progenitor cells," Cell Cycle, vol. 5, no. 3, pp. 327-335, 2006.

[4] D. Padfield, J. Rittscher, and B. Roysam, "Coupled minimum-cost flow cell tracking," Information Processing in Medical Imaging, pp. 374-385, 2009.

[5] R. Bise, K. Li, S. Eom, and T. Kanade, "Reliably tracking partially overlapping neural stem cells in dic microscopy image sequence," MICCAI Workshop, pp. 67-77, 2009.

[6] D. Reid, "An algorithm for tracking multiple targets. IEEE Trans," Automatic Control 24, 843-854, 1979.

[7] T. Fortmann, Y.B. Shalom, and M. Scheffe, "Sonar tracking of multiple targets using joint probabilistic data association,” IEEE J. Oceanic Engineering 8, 173-184, 1983.

[8] C. Huang, B. Wu, and R. Nevatia, "Robust Object Tracking by Hierarchical Association of Detection Responses," ECCV, p788-801, 2008.

[9] H.W. Kuhn, "The Hungarian method for the assignment problem,' Naval Research Logistics Quarterly 2, 83-87, 1955.

[10] S. Bonneau, M. Dahan, L.D. Cohen, "Single quantum dot tracking based on perceptual grouping using minimal paths in a spatiotemporal volume," Image Processing, 14, 9, 1384 - 1395, 2005.

[11] L. Zhang, Y. Li, and R. Nevatia, "Global data association for multi-object tracking using network flows," CVPR, 2008.

[12] Z. Yin, K. Li, T. Kanade, and M. Chen, "Understanding the Optics to Aid Microscopy Image Segmentation," MICCAI, 2010.

[13] S. Huh, E.D.F Ker, R. Bise, M. Chen, and T. Kanade, "Automated mitosis detection of stem cell populations in phase-contrast microscopy images," IEEE Trans.Med. Imag, In press.

[14] S. Blackman, "Multiple-target tracking with radar applications," Artech House Publishersl, 1986. 